\section{Magic clusters}

Nano Lett. doi:10.1021/nl801685a (2008)

Semiconductor nanocrystals have led to extraordinary applications, ranging from biomedical to efficient electronic devices. But now there are even smaller nanoparticles that may one day outperform their larger relatives: magic-sized clusters. Experiments on clusters of CdSe have so far only achieved very poor optical properties. Christopher Evans and colleagues have instead focused on PbSe. They followed a similar route to that used to synthesize $\mathrm{PbSe}$ nanocrystals, that is, heating $\mathrm{PbO}$ and oleic acid at $150^{\circ} \mathrm{C}$ in octadecene. But this time they cooled down the solution and let it settle at $20^{\circ} \mathrm{C}$ for several hours. The absorption spectrum of the solution measured at regular intervals shows the appearance and subsequent disappearance of peaks at well-defined wavelengths, indicating the formation of clusters with well-defined size and their subsequent transformation into larger ones. These clusters have a very efficient optical emission in the near infrared, which is ideal for in vivo fluorescence applications. In addition, their small diameter $(<2 \mathrm{~nm})$ would allow their transport through cells, making them a strong potential fluorophore for biological imaging.

\section{Express delivery}

J.Am. Chem. Soc. doi:10.1021/ja801631c (2008) Photodynamic therapy (PDT) is a promising method for treating several cancers that uses photosensitizers. The photosensitizer accumulates in target tissue and is excited using light, generating reactive oxygen species that induce cell death. To enter the target area PDT drugs need to be lipophilic, however this presents problems: lack of solubility in the physiological conditions experienced en route to the target means that they usually take at least 24 hours to get to the tumour. Baowei Fei, Clemens Burda and co-workers have synthesized a PDT drug complex that significantly decreases the delivery time and enables quantitative monitoring of the process. Polyethylene glycol (PEG; a compound approved for human intravenous applications) is attached to gold nanoparticles functionalized with the PDT drug, silicon phthalocyanine-4. Chains of PEG wrap around the drug allowing the system to disperse in aqueous conditions. Free silicon phthalocyanine- 4 takes $1-2$ days to accumulate in a tumour site; using the new complex this is reduced to less than two hours in vivo in cancer-bearing mice with the tumour reducing in size within one week of treatment.

\section{Helical swirls}

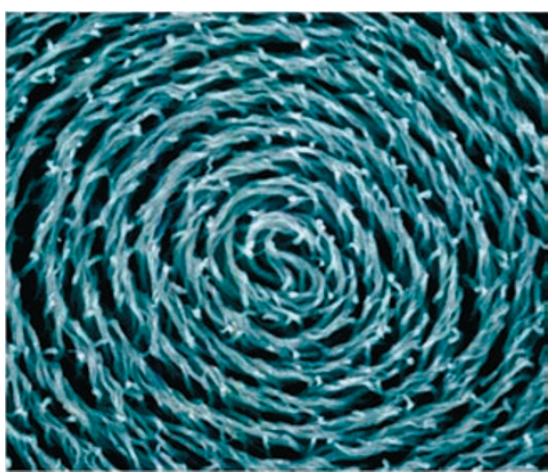

J.Am. Chem. Soc. doi.10.1021/ja803865e (2008) It is well known that iodine-doping of polyacetylene films dramatically increases the electrical conductivity of the material. Now, Mutsumasa Kyotani, Kazuo Akagi and colleagues have discovered another benefit - it prevents the polymer film from thermally decomposing at high temperatures. Helical nanofibres of polyacetylene that are twisted and concentrically curled to form a swirl do not retain their morphology at high temperatures. However, if iodine is added to the polymer it is possible to preserve this morphology on heating and make carbon and graphitic films. The carbon film is made by heating the iodinedoped polyacetylene to $800{ }^{\circ} \mathrm{C}$ and, if this twisted bundle of carbon nanofibres is heated further to $2,600{ }^{\circ} \mathrm{C}$, graphitic crystallization occurs, which results in a graphitic film. The iodine-doped

\section{Totally smooth}

\section{Adv. Mater.}

doi:10.1002/adma.200800866 (2008)

Helium atom scattering is a powerful technique for probing surfaces that uses low energies, and has the advantage that it does not affect the surface. By focusing a beam of He atoms into a small spot, a scanning helium atom microscope could then be used as an imaging probe for particularly delicate samples. An ideal focusing material would be an ultraperfect semiconductor wafer covered by a nanometre-thick metal film. However, when metals are deposited on semiconductors they form islands of irregular height, adversely affecting the $\mathrm{He}$ scattering. Now Daniel Barredo and coworkers have synthesized $\mathrm{a} \mathrm{Pb}-\mathrm{Si}$ material that specularly reflects the incoming beam allowing it to act as a focusing mirror for neutral atoms. The researchers exploit a quantum-confinement effect: if metals are deposited on a substrate with an absolute polyacetylene is not stable under atmospheric conditions, but the carbon and graphitic films are, and hence are likely to find practical use in the many applications for which carbon films show promise, including electrochemical energy storage, cell electrodes and gasabsorbent materials.

\section{Morphological control}

Chem. Mater. doi.10.1021/cm801028a (2008)

The porosity and internal structure of conducting polymer nanowires strongly influences their transport properties and device performance. However, ways of controlling their morphology without using templates have so far been limited. Thomas Mallouk and Yanyan Cao now report the electrochemical growth of polyaniline nanowires from aqueous sulphuric acid solutions in anodic aluminium oxide membranes. Increasing the sulphuric acid concentration during growth induces a transition from solid to open nanowires. The electrochemical capacitance of these resulting nanowire arrays is also shown to be directly related to their morphology. At high anodic potential the nanowires exhibit a porous morphology whereas at a lower potential they show a dense structure consisting of interconnected fibres. Overall, porosity increases the specific capacitance, and this approach to produce these polynaniline nanowires with controlled morphology should prove useful for device applications such as electrochemical supercapacitors.

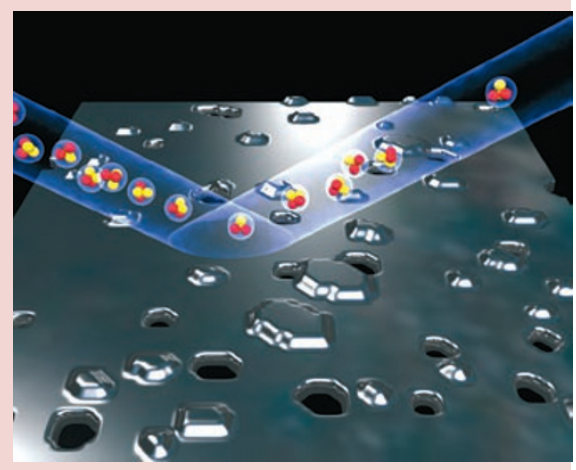

gap around the Fermi energy, electrons from the metal are strongly electronically confined. Consequently, some metal heights are more stable than others, thus producing a uniform film. The films become more atomically flat on heating, resulting in a reflective film with the same perfection as the $\mathrm{Si}(111)$ substrate. 